\title{
Chronotherapy for morning blood pressure surge in hypertensive patients: a systematic review and meta-analysis
}

\author{
Ziyan Xie, Jiahao Zhang, Chenyu Wang and Xiaowei Yan* (1)
}

\begin{abstract}
Background: The morning blood pressure surge (MBPS) is related to an exaggerated risk of cardiovascular diseases and mortality. With increasing attention on circadian change in blood pressure and extensive use of ambulatory blood pressure monitoring (ABPM), chronotherapy that administration of medication according to biological rhythm, is reported to improve cardiovascular outcomes. The aim of this study is to evaluate the influence of chronotherapy of antihypertensive drugs upon MBPS in hypertensive patients.

Methods: A search strategy was applied in Ovid MEDLINE, EMBASE, Cochrane (Wiley) CENTRAL Register of Controlled Trials, Cochrane Database of Systematic Reviews, and the Chinese Biomedical literature database. No language and date restrictions. Randomized controlled trials (RCT) assessing the efficacy of evening and morning administration of the same medications in adult patients with primary hypertension were included.

Results: A total of ten trials, comprising 1724 participants with a mean age of 61 and 51\% female, were included in this study. Combined analysis observed significant reduction of MBPS $(-5.30 \mathrm{mmHg}, 95 \% \mathrm{Cl}-8.80$ to -1.80$)$, night-time SBP $(-2.29 \mathrm{mmHg}, 95 \% \mathrm{Cl}-4.43$ to -0.15$)$, night-time DBP $(-1.63 \mathrm{mmHg}, 95 \% \mathrm{Cl}-3.23$ to -0.04$)$ and increase in night blood pressure dipping $(3.23 \%, 95 \% \mathrm{Cl} 5.37$ to 1.10$)$ in evening dosage compared with traditional morning dosage of blood pressure-lowering drugs. No significant difference was found in the incidence of overall adverse effects (RR $0.65,95 \% \mathrm{Cl} 0.30$ to 1.41) and withdrawal due to adverse effects (RR $0.95,95 \% \mathrm{Cl} 0.53$ to 1.71 ).

Conclusions: Our study suggested that evening administration of antihypertensive medications exerted better blood pressure-lowering effect on MBPS compared with conventional morning dosage. Safety assessment also indicated that the evening regimen did not increase the risk of adverse events. However, endpoint studies need to be carried out to confirm the significance and feasibility of this treatment regimen in clinical practice.
\end{abstract}

Keywords: Morning blood pressure surge, Circadian rhythm, Chronotherapy, Administration time, Hypertension

\section{Background}

The circadian rhythm plays a critical role in multiple neurohormonal processes, thus modulating the cardiovascular system [1,2]. The circadian changes in blood pressure have received increasing interest. Additionally, with the

\footnotetext{
*Correspondence: yanxw@pumch.cn

Department of Cardiology, Peking Union Medical CollegeHospital, Peking

Union Medical College \& Chinese Academy of Medical Sciences, No.1

ShuaiFuYuan, Beijing 100730, China
}

introduction of ambulatory blood pressure monitoring (ABPM) into regular hypertension management, daynight patterns of blood pressure can be observed [3]. Early morning is the time of the highest incidence of cardiovascular events during the day [4]. Accumulating evidence demonstrated that an exaggerated morning blood pressure surge (MBPS) is closely related to the increased risk of cardiovascular diseases and all-cause mortality [5-8]. Besides, systematic review also indicated that 
when using a continuous variable to test correlations, a $10 \mathrm{~mm} \mathrm{Hg}$ increase in MBPS was related to high risk of stroke [9]. Thus, the control of MBPS is clinically relevant and important. In addition, night blood pressure dipping, an index of blood pressure decline, has also been reported as an important prognostic marker for cardiovascular events and morbidity in hypertensives $[10,11]$.

Chronotherapy is the adaption of medication to biological rhythm, to achieve maximal effectiveness by altering the time of drug administration [12], which could influence the pharmacokinetic properties of antihypertensive medications. Multiple works have evaluated the administration-time effects in hypertension therapy. Hermida et al. revealed that administration of at least one prescribed antihypertensive medications in hypertensives at bedtime, compared to upon wakening, significantly improved blood pressure control (especially bedtime blood pressure) and remarkably diminished the occurrence of major cardiovascular events, such as stroke, myocardial infarction, death, etc. [13]. A Cochrane systemic review showed that bedtime ingestion of antihypertensive drugs was more effective to decrease 24-h blood pressure without additional adverse effects than morning regimen [14].

Some studies documented that bedtime ingestion of blood pressure-lowering agents had a more efficient antihypertensive effect during night-time and at the early morning period [15-17]. Most studies working on the administration time of antihypertensive medications were small-scaled and single-centered. And the bedtime administration has not been recommended in guidelines. Whether the bedtime administration of antihypertensive medications has a more significant effect on lowering MBPS than conventional morning administration has not been reported in any systematic review and metaanalysis. Therefore, this systematic review was conducted to investigate the efficacy of chronotherapy of once-daily antihypertensive drugs on reducing MBPS, systolic and diastolic blood pressure, and adverse effects in hypertensive patients, thus providing more evidence to the bedtime regimen.

\section{Methods}

The protocol for this systematic review and meta-analysis was registered in PROSPERO (CRD42020180166).

\section{Search strategy}

The searching databases included: Ovid MEDLINE, EMBASE, Cochrane (Wiley) CENTRAL Register of Controlled Trials and Cochrane Database of Systematic Reviews, the Chinese Biomedical literature database up to April 2020. Besides, we also tracked completed clinical trials that met our inclusion criteria on ClinicalTrials.gov.
The major search terms included: "hypertension", "morning surge", "morning blood pressure surge", "MBPS", "night" decline", "Chronotherapy", "morning OR day OR am OR diurnal OR daytime OR awake", "evening OR bedtime OR night OR nocturnal OR pm" (shown in Additional file 1: Table 1). In addition to databases, studies in references lists of relevant articles that met our criteria were also hand-searched and screened as a significant supplement. No language and date restrictions were applied to avoid missing any related investigations.

\section{Inclusion and exclusion criteria}

Study selection was performed independently by two reviewers (Z.X. and J.Z.) by viewing the titles and the abstracts of the search strategies. The disagreements were judged by a third reviewer who was blinded to the first two reviewers' decisions. The selection process was repeated twice by each reviewer. Studies meeting the inclusion criteria were checked in detail (full text).

Only randomized control trials (RCT) were included to assess the effects of chronotherapy. Randomized crossover trials that only had two treatment periods (two interventions) were also considered. Adults (more than 18) with primary hypertension, which is defined as systolic and/or diastolic blood pressure levels more than 140/90 mmHg were included. Secondary hypertension, alternating shift workers, and severe cardiac insufficiency (NYHA III-IV) were the exclusion criteria. Studies reporting one-drug therapy or combined therapy (two or more drugs) with the antihypertensive drug(s) administered once daily at bedtime when the control group was the same $\operatorname{drug}(\mathrm{s})$ at the same dose(s) once a day upon awakening were included. Antihypertensive drugs comprised diuretics, adrenergic beta-antagonists ( $\beta$-blockers), alpha-antagonists, calcium channel blockers (CCB),vasodilator agents and renin-angiotensin system inhibitors (RASI, including angiotensin II receptor blockers and angiotensin-converting enzyme inhibitors). Administration time in the evening was from 18:00 to 24:00, while in the morning was from 6:00 to 12:00.

Included studies must clearly define and measure MBPS. Both continuous and categorical variables were included. Definitions of MBPS must belong to any one of the following: (1) the sleep-trough surge, calculated by the mean value of morning blood pressure within $2 \mathrm{~h}$ after waking minus the lowest night-time blood pressure; (2) the prewaking surge, the mean blood pressure within $2 \mathrm{~h}$ after waking to subtract the mean blood pressure within $2 \mathrm{~h}$ before waking; (3) the rising blood pressure surge, morning blood pressure reading upon rising minus blood pressure reading in the lying position $30 \mathrm{~min}$ before rising. The measurement of night dipping is the 
percentage of reduction in mean nighttime blood pressure relative to mean daytime blood pressure.

\section{Outcomes}

The primary outcome was the change in MBPS from baseline to the end of treatment (or the value of MBPS at the end of treatment if the baseline is comparable) when it was calculated as a continuous variable; or the ratio of patients whose MBPS exceeded the settled threshold after treatment when MBPS was defined as a categorical variable.

The secondary outcomes contained night blood pressure dipping, 24-h mean systolic blood pressure (SBP) and diastolic blood pressure (DBP), daytime mean SBP and DBP (measured by ABPM from the time patients wake up in the morning to the time they fall asleep in the evening or from 6-8:00 to 22-24:00), night-time SBP and DBP (measured by ABMP from the time patients fall asleep in the evening to the time they wake up in the morning or from 22-24:00 to 6-8:00). Safety outcomes include overall adverse effects and withdrawals due to adverse effects during treatment. Adverse effects can be any unplanned and unfavorable symptom, or disease temporally associated with the use of medicine. Withdrawals due to adverse effects are reported as events leading to permanent trial discontinuation.

\section{Data extraction and management}

Data were extracted by two independent reviewers (Z.X. and J.Z.) based on a standard form. Necessary information of studies was extracted, such as patients' demographics (age, sex, region, race, hypertensive status, medication history), study methods (patients' recruitment, randomization, crossover, treatment duration), interventions (drugs, dose, administration time), outcomes (definition and measurement of MBPS, night blood pressure dipping, 24-h mean SBP/DBP, daytime mean SBP/DBP, night-time SBP/DBP) and safety endpoints (headache, nasopharyngitis, edema, bronchitis, pain and withdrawals due to adverse effects). The numeric data (e.g., blood pressure) were collected from text and tables. Data in graphs were not extracted due to a possible measurement error. All the studies were double coded by the two reviewers. If there were missing data, we attempted to contact the authors to provide the missing information. Regarding missing data for the standard deviation of the change in MBPS, imputation was conducted based on other similar trials.

\section{Risk of bias assessment}

Evaluation of the risk of bias in all included trials was executed by two independent reviewers (Z.X. and J.Z.) based on the guide of the Cochrane risk of bias tool.
Characteristics of assessment included random sequence generation, allocation concealment, blinding, incomplete outcome data, selective reporting, and other bias (e.g., conflict of interest). The assessment was done at the study level.

\section{Data analysis}

All data analysis and synthesis in the meta-analysis was conducted by RevMan 5.4 (RevMan 5.4; Cochrane Collaboration, Oxford, UK). Comparisons of continuous MBPS changes, 24-h, daytime and night-time SBP and DBP between groups were presented as mean \pm standard deviation (SD) with corresponding 95\% confidence intervals. These data were entered using a generic inverse variance. Categorical MBPS, overall adverse effects, and withdrawals were presented as relative risk ratios (RR). The outcomes were combined using a fixed- effect model $\left(\mathrm{I}^{2} \leq 25 \%\right)$ and a random-effect model $\left(\mathrm{I}^{2}>25 \%\right)$.

\section{Subgroup analysis}

Subgroup analysis were conducted by classifying the trials into those using different MBPS definitions, and those using antihypertensive drugs from different classes. Subgroup analysis can help locate the sources of heterogeneity and assess the effectiveness of different kinds of medicines.

\section{Results}

\section{Search results}

636 records were detected after database searching, and 4 additional articles were hand-searched in reference lists of published papers. After the removal of duplicates, 569 articles were viewed by titles and abstracts by two independent reviewers (Z.X. and J.Z.), 401 of which were excluded, and the remaining 168 references entered full-text screening for intensive evaluation. Eventually, 10 articles (10 trials) were considered for analysis and involved in our study. The reasons for excluding 158 references are shown in Fig. 1.

\section{Description of included studies}

Included studies were 8 parallel-designed RCTs and 2 crossover-designed RCT [18] with 1724 participants in 8 different countries. The key characteristics of the trials are listed in Table 1 . The study size ranged from 31 to 639 . The mean age of patients was 61 years, and females accounted for $43-61 \%$. The duration of followup periods differed from studies (ranged from 2 to 48 weeks). Eight studies described ethnicity. One trial was conducted in the USA, with 38 participants (2\%), half of which were African American [18]. One trial (37\%) was a multi-center study conducted in 94 centers in five countries with nearly $99 \%$ white [19]. Six studies 


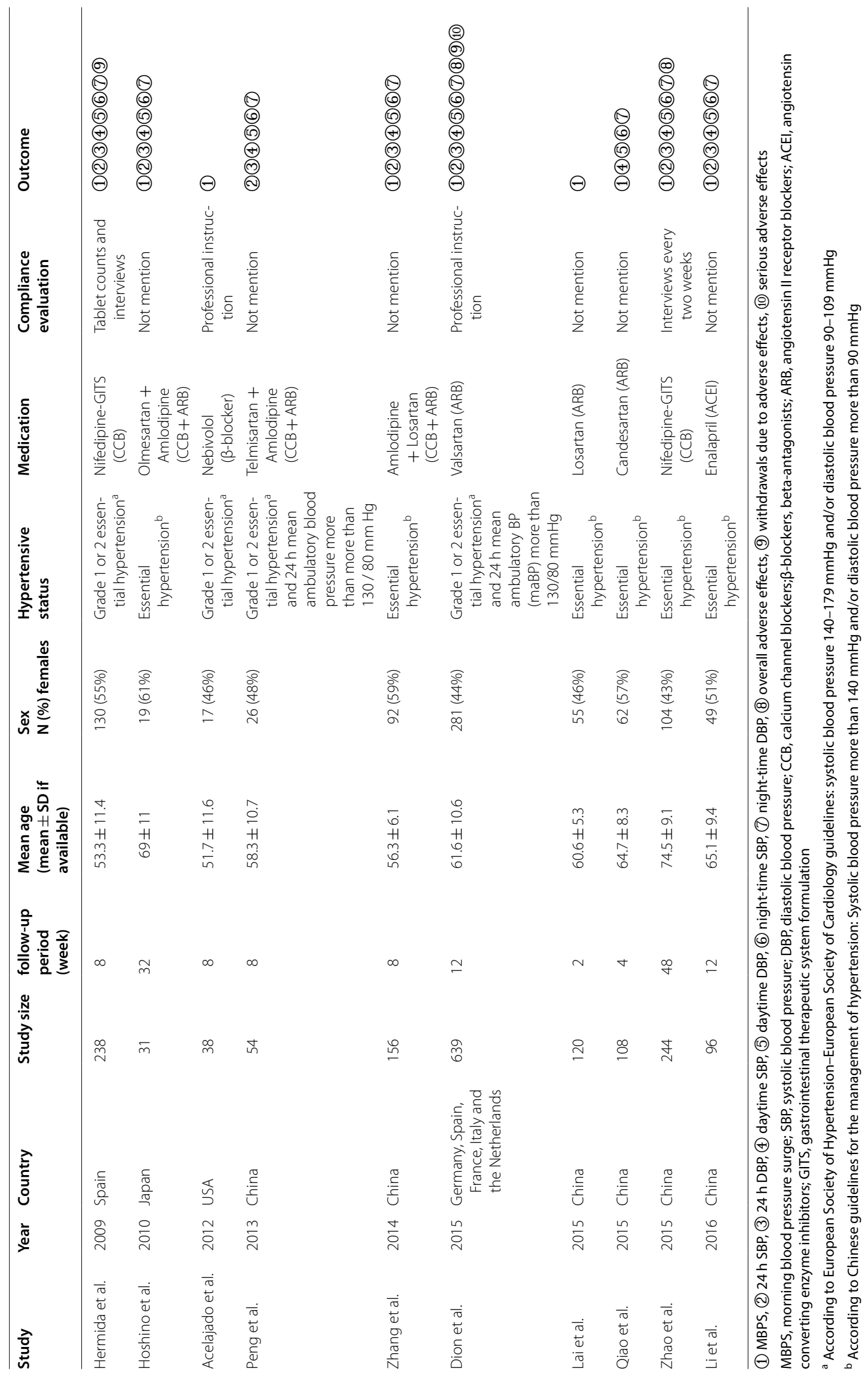




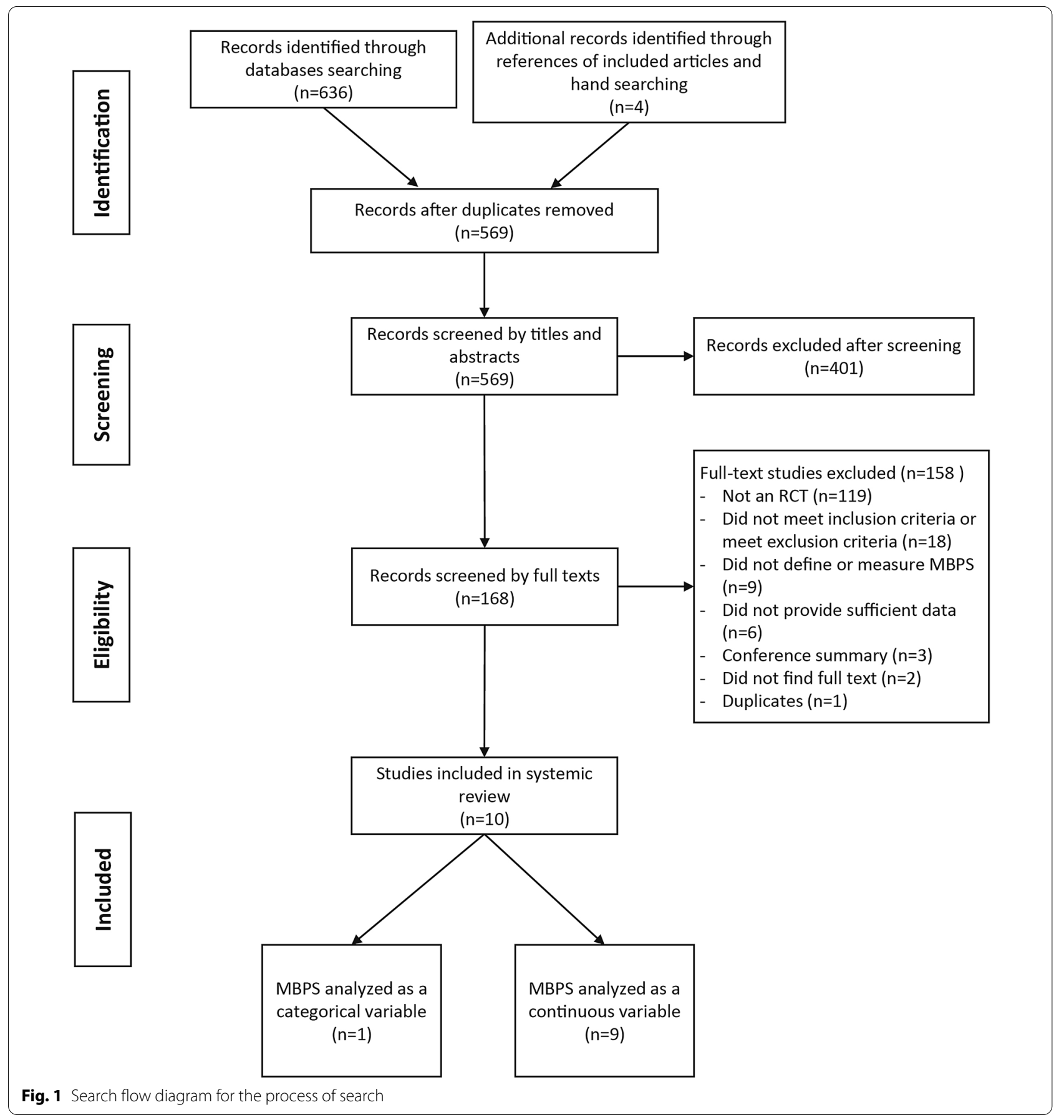

with 778 (45\%) participants were recruited from China [20-25]. The remaining two trials without reporting race were organized in Europe (14\%) and Japan (2\%) [26]. The definitions and measurements of MBPS were listed in Additional file 1: Table 2. Peng et al. analyzed MBPS as a categorical variable [25], and the study of Zhang et al. contained both continuous and categorical variables [20]. Among all the trials, seven studies presented secondary outcomes [19-23, 25, 26].

\section{Risk of bias in the included studies}

The overall assessment of the risk of bias in the included studies are shown in Additional 1: Table 3. One study, as a crossover RCT, only the first period data were available 
[18]. One study had the risk of baseline imbalance [22]; six studies provided insufficient information about other bias [19, 21, 23-25]. Good random sequence generation, concealed allocation and blinding were ascertained in all the studies. And none of the trials had attrition bias.

\section{Effects of interventions}

\section{Changes in MBPS}

The most common definition of MBPS (sleep-trough surge) were compared in included studies (Fig. 2). The overall analysis showed that the evening administration significantly decreased MBPS by $5.30 \mathrm{mmHg}(95 \%$ $\mathrm{CI}-8.80$ to -1.80$)$. But a significant heterogeneity $\left(\mathrm{I}^{2}=98 \%\right)$ was detected.

The subgroup analysis observed significant differences in the evening dosage regimen of CCB and RASI compared with morning administration. The evening administration of CCB lowered MBPS by $4.21 \mathrm{mmHg}$ (95\% CI -4.66 to -3.76 ), and RASI reduced MBPS by $6.90 \mathrm{mmHg}(95 \% \mathrm{CI}-11.49$ to -2.31$)$. No heterogeneity $\left(\mathrm{I}^{2}=0 \%\right)$ was observed in the $\mathrm{CCB}$ subgroup, while high heterogeneity $\left(\mathrm{I}^{2}=97 \%\right)$ was detected in the RASI subgroup. No differences in blood pressure reduction were observed with $\beta$-blockers $(P=0.67)$ and combined
$\mathrm{CCB}$ and $\mathrm{ARB}(P=0.52)$ in the evening versus morning administration.

The effects of intervention were also analyzed when MBPS was measured as categorical variable (Additional file 1: Fig. 1). No significance was detected in overall analysis. Due to the low number of trials analyzing MBPS as a categorical variable and different thresholds of MBPS, it might be insufficient to carry out a combined analysis.

\section{Changes in SBP}

The data and results of SBP are presented in Fig. 3. The overall effects of evening administration showed no statistically significant reduction in 24-h SBP $(P=0.27)$ and daytime SBP $(P=0.78)$. However, evening regimen significantly decreased night-time SBP by $2.29 \mathrm{mmHg}(95 \%$ CI -4.43 to -0.15$)$, compared with morning administration. The subgroup analysis (Additional file 1: Table 4) showed that $\mathrm{CCB}$ evening regimen significantly reduced 24-h SBP, daytime SBP and night-time SBP by $4.1 \mathrm{mmHg}$ (95\% CI -5.28 to $\left.-2.92, \mathrm{I}^{2}=0 \%\right), 3.72 \mathrm{mmHg}(95 \% \mathrm{CI}$ -5.04 to $\left.-2.39, \mathrm{I}^{2}=0 \%\right)$ and by $5.37 \mathrm{mmHg}(95 \% \mathrm{CI}$ -6.92 to $\left.-3.82, \mathrm{I}^{2}=0 \%\right)$, respectively. There were no significant differences of SBP reduction were detected in RASI and CCB + ARB combined therapy.

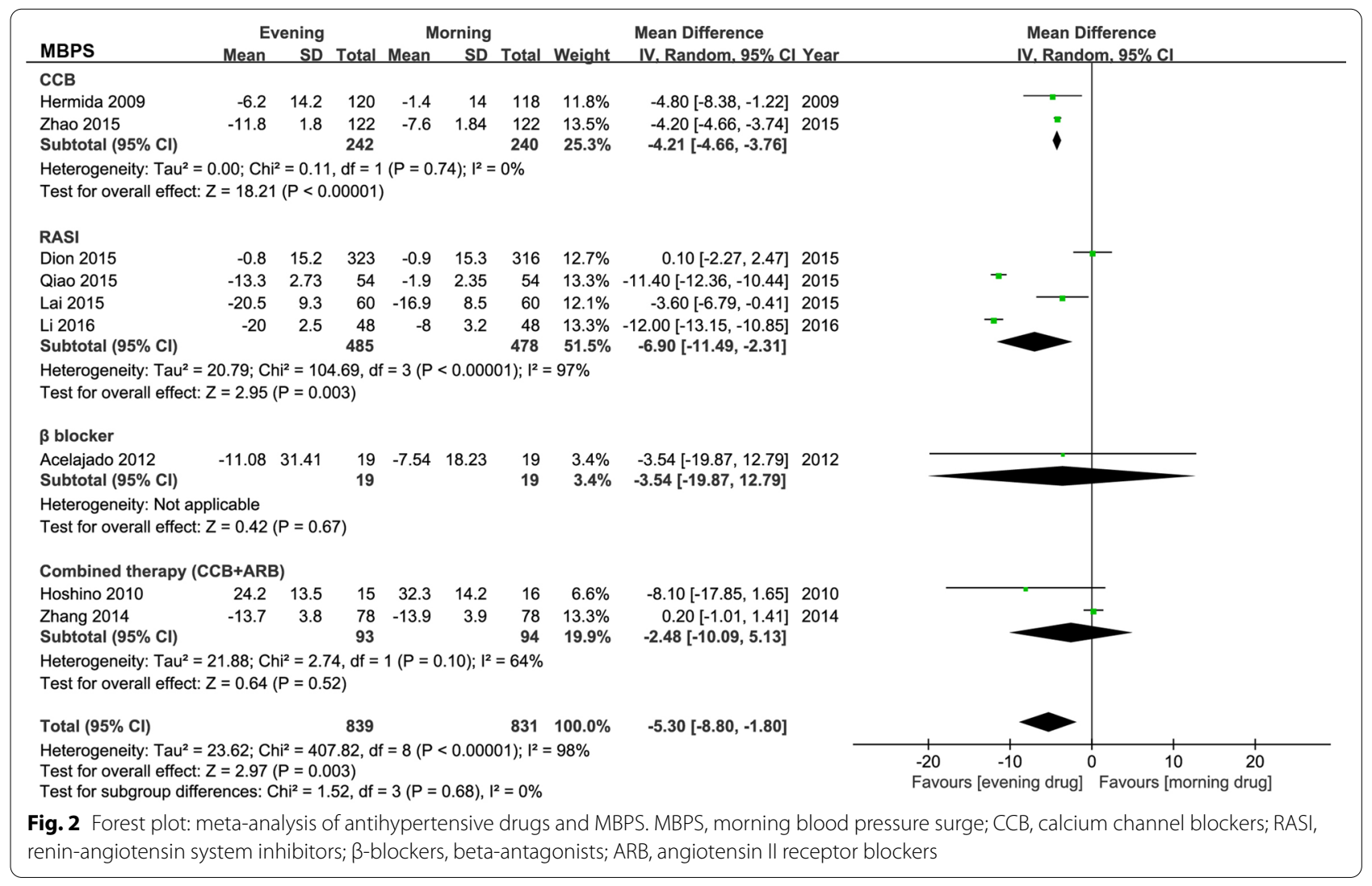




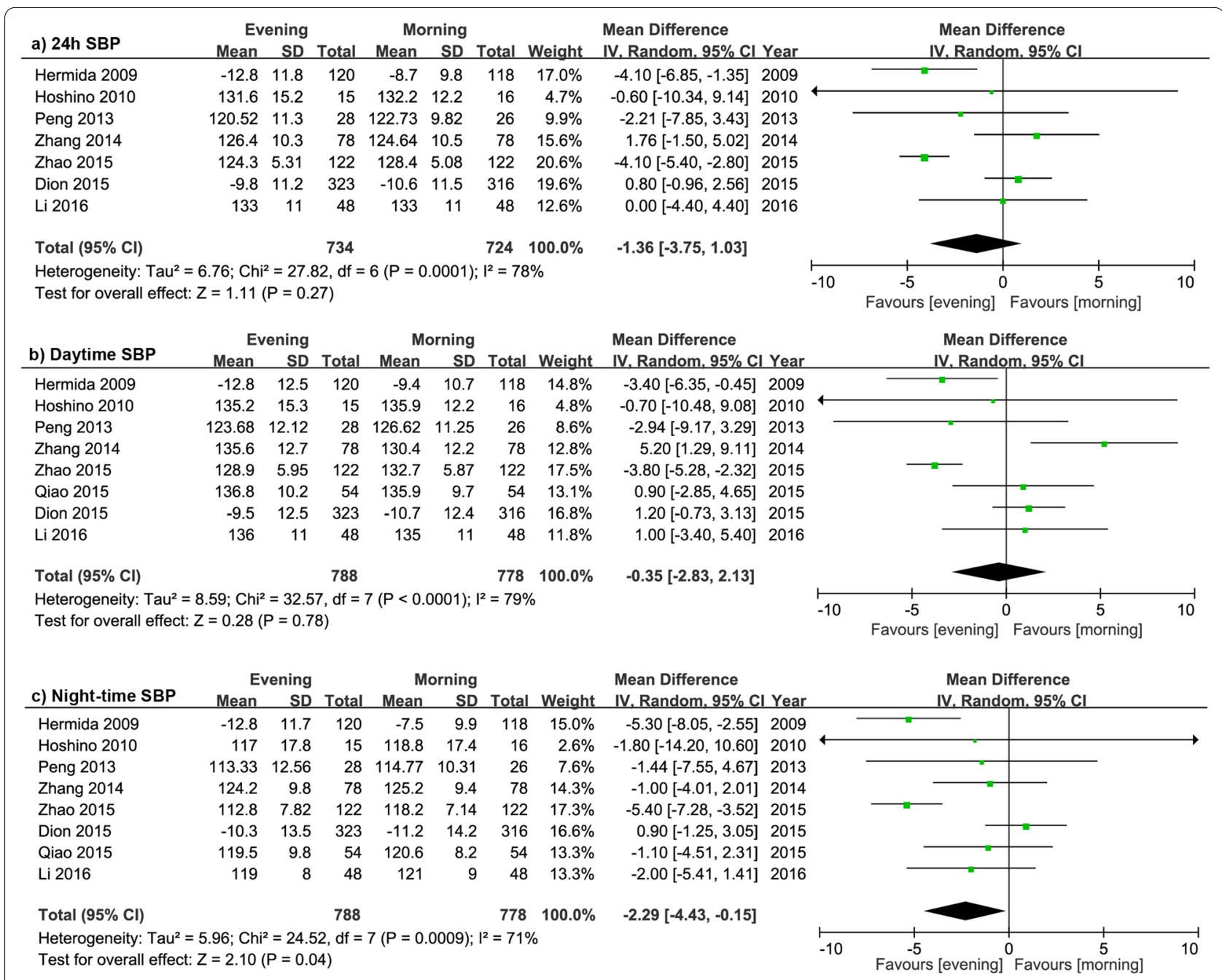

Fig. 3 Forest plot: evening versus morning dosing regimen in $24 \mathrm{~h}$ SBP, daytime SBP and night-time SBP. SBP, systolic blood pressure; DBP, diastolic blood pressure

\section{Changes in DBP}

The details of efficacy in DBP are shown in Fig. 4. The analysis of overall effects demonstrated that evening dosage regimen significantly reduced night-time DBP by $1.63 \mathrm{mmHg}(P=0.04)$ and no significant differences were observed in 24-h DBP $(P=0.31)$, daytime DBP $(P$ $=0.35$ ). The subgroup analysis (Additional file 1: Table 4 ) showed that the evening $\mathrm{CCB}$ was associated with significant 24-h DBP and night-time DBP reduction by $3.32 \mathrm{mmHg}(95 \% \mathrm{CI}-5.85$ to -0.78$)$ and $3.81 \mathrm{mmHg}$ (95\% CI -5.45 to -2.18$)$, respectively; and no significant differences of DBP reduction were found in RASI or $\mathrm{CCB}+\mathrm{ARB}$ combined therapy subgroups.

\section{Changes in night BP dipping}

6 studies with 3674 hypertensive subjects were included and analyzed. The result indicated that bedtime dosing remarkably enhanced nocturnal blood pressure decline by $3.23 \%$ ( $95 \%$ CI 5.37 to 1.10), compared with morning dosage regimen (Additional file 1: Fig. 2). And a high heterogeneity $\left(\mathrm{I}^{2}=89 \%\right)$ was detected.

\section{Adverse effects}

The meta-analysis showed that the incidence of overall adverse effects (RR $0.65,95 \%$ CI 0.30 to $1.41, \mathrm{I}^{2}=69 \%$ ) and discontinuations due to adverse effects (RR 0.95, 95\% CI 0.53 to $1.71, \mathrm{I}^{2}=0 \%$ ) had no significant differences between evening and morning regimens (Additional file 1: Fig. 3 and Table 5). Among all the kinds of adverse effects reported, headache was the most frequently reported symptom, and a slightly significant increase of nausea was observed in the morning regimen $(P=0.05)$. 


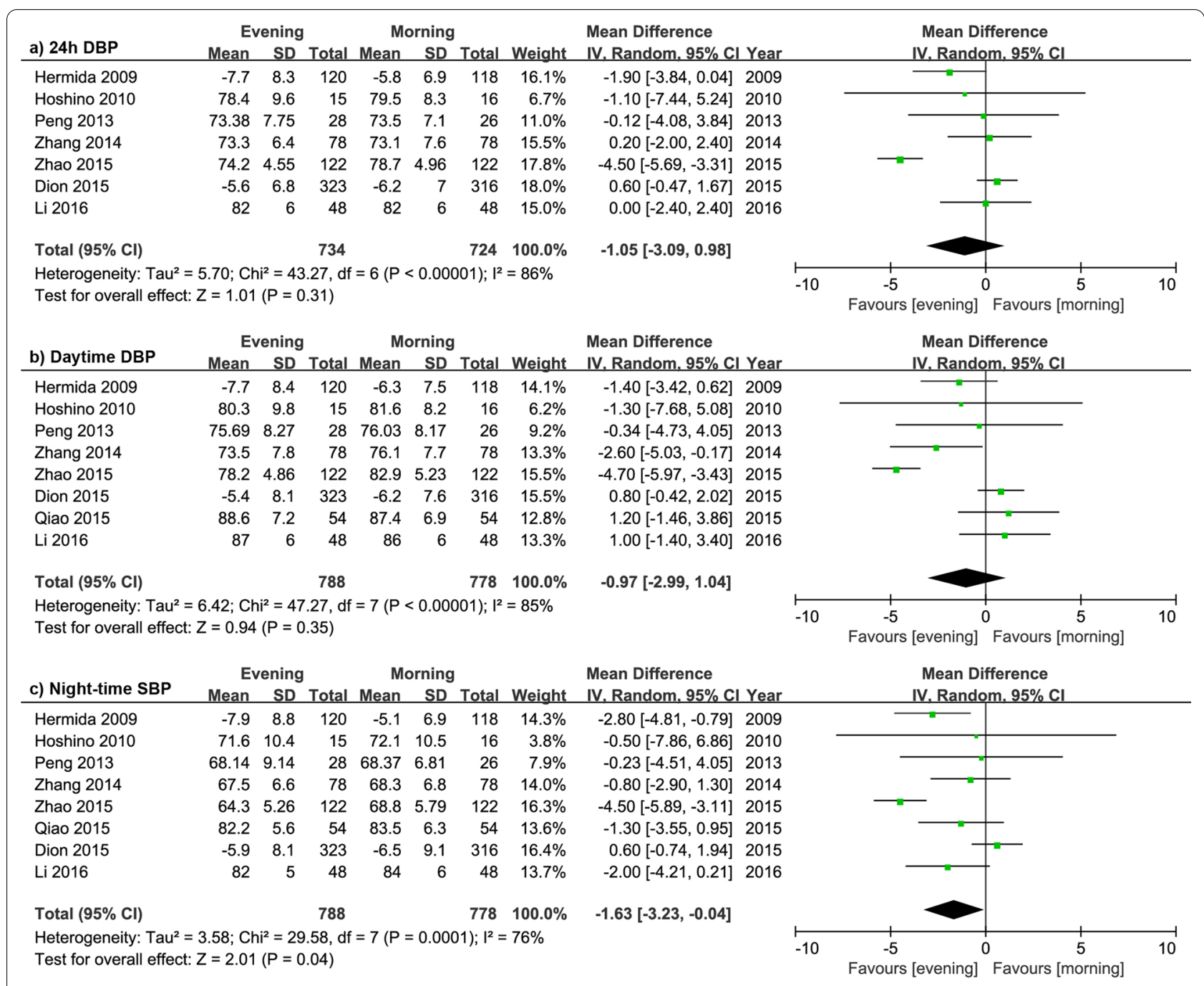

Fig. 4 Forest plot: evening versus morning dosing regimen in $24 \mathrm{~h} \mathrm{DBP}$, daytime DBP and night-time DBP. DBP, diastolic blood pressure

\section{Discussion}

This systematic review and meta-analysis investigated the effects of evening and morning dosage of antihypertensive medications on MBPS and blood pressure control in patients with essential hypertension.

Morning administration of medication is commonly used in antihypertensive treatment in clinical practice. However, some recent clinical trials reported evening administration of the antihypertensive drug to be efficient in lowering blood pressure, improving organ functions, and preventing cardiovascular events [27-30]. This study indicated that the evening regimen of antihypertensive drugs exerted better effects on decreasing night-time blood pressure than morning therapy. Since studies showed that night-time blood pressure was significantly better than daytime blood pressure in predicting all-cause, cardiovascular and non-cardiovascular mortality, stroke and cardiovascular events [31, 32]. Besides, this analysis also demonstrated that bedtime administration significantly better enhanced night blood pressure dipping, relative to morning regimen, which was consistent with several previous studies. Increased nocturnal decline drives blood pressure towards more of a dipper pattern, thus improving blood pressure variability and lowering cardiovascular risk $[33,34]$. Therefore, chronotherapy targeting night-time blood pressure control may favorably affect cardiovascular morbidity and mortality in hypertensive patients.

This study found that evening administration of blood pressure-lowering medication significantly improved MBPS, which has not been systematically reviewed before. The increase of MBPS was first associated with 
the incidence of stroke in 2003 [8]. A clinical trial with 519 hypertensive participants found that a $10 \mathrm{~mm} \mathrm{Hg}$ raised in MBPS caused a $25 \%$ increased stroke incidence. Several but not all subsequent studies indicated that exaggerated MBPS had been related to increased risk for cardiovascular events and all-cause mortality. However, in recent years, the prognostic role of MBPS is controversial.

One possible reason is the distinct definitions and cut-off points of MBPS. There are four definitions commonly used (the sleep-trough surge, the prewaking surge, the rising blood pressure surge, and the morning-evening difference), and the thresholds are various. The thresholds of MBPS could be defined as top decile, quartiles of MBPS in participants, or determined according to hypertensive guidelines, which have not reached a consensus [35]. Among included studies, two articles analyzed MBPS as categorical variable with different predetermined threshold, $23.58 \mathrm{mmHg}$ and $35 \mathrm{mmHg}$, respectively. Due to the limited number and different thresholds of studies, the heterogeneity between studies of categorical MBPS is very high. And a previous metaanalysis found that there was no significant association between MBPS and all-cause mortality or cardiovascular events when MBPS was analyzed as categorical variable [9]. Therefore, a single threshold dichotomizing MBPS as normal and exaggerated may not be powerful to define the significance of MBPS. Besides, in patients with nondipper hypertension and nocturnal hypertension, which are described as a sleep-to-awake SBP ratio of less than $10 \%$, and night-time SBP more than $120 \mathrm{mmHg}$ and DBP more than $70 \mathrm{mmHg}$ respectively [36], the MBPS may be low and not suitable for the evaluation of cardiovascular prognosis. This was also supported by the Jackson Heart Study, that no significant association between MBPS and the incidence of cardiovascular events was found in the black population with non-dipper and nocturnal hypertension [37]. Consequently, more research is needed to analyze MBPS's definitions and reach a consensus on the target value of MBPS control. In patients with nocturnal dipping hypertension, a prognostic indicator needs to be developed to predict cardiovascular events and serve as a target of hypertension management.

On the other hand, ethnicity is a critical factor affecting morning blood pressure surge. The Ohasama Study [7] and JMS-ABPM Study [8] found significant relationship between exaggerated MBPS and stoke incidence in Japanese patients. Conversely, Bombelli et al. [38] reported that high MBPS was not associated with increased mortality and cardiovascular events in a white population. Furthermore, systematic review showed that the degree of sleep-trough surge was higher in Japanese than in European patients with hypertension [39]. The Jackson
Heart Study also revealed that there was no clear evidence for the associations of sleep-trough MBPS, prewaking MBPS, and rising blood pressure surge with the incidence of cardiovascular events and all-cause mortality in black adults [37]. Discrepancies in the pathogenesis and manifestations of hypertension and related cardiovascular outcomes have been consistently reported among different races. Asians have more active sympathetic nerve activity during the morning period and higher incidence of stroke, which may account for the high degree and the prognostic role of MBPS [4]. Therefore, therapeutic strategies targeting MBPS control are of considerable significance in Asian population.

This meta-analysis discovered a prominent role of evening $\mathrm{CCB}$ in lowering blood pressure. The CCBs in included studies were nifedipine gastrointestinal therapeutic system (GITS) and amlodipine, which both belongs to the dihydropyridine calcium blocker family with long duration of action [40]. Amlodipine has been shown to be more effective for the MBPS control compared with valsartan in the VALUE trial [41]. Besides, previous evidence observed that the pharmacokinetic pharmacodynamics relationship of CCB is circadian rhythm-dependent that a night dosing of amlodipine displayed a longer half-life and higher peak plasma concentration compared to the morning dosage $[42,43]$. This may also explain the remarkable effects of CCB in lowering night-time and MBPS when given at bedtime.

\section{Limitations}

This study has some potential limitations. First, the searching results indicated that only ten studies were eligible for combined analysis, and six of them were conducted in China, which reflects the racial differences in the attention to MBPS. The lack of evidence also posed a limitation to the analysis of distinct definitions and thresholds of MBPS and sensitivity analysis according to methodological quality. Besides, the blood pressure-lowering efficacy alone is not adequate to judge the clinical significance of antihypertensive of chronotherapy. Thus long-term, larger scale and multi-racial clinical trials are needed to explore the correlation between MBPS reduction and the incidence of cardiovascular endpoint.

\section{Conclusions}

MBPS has received considerable attention from clinicians. Our meta-analysis provided the evidence that evening administration of antihypertensive medication improves blood pressure variability by significantly reducing MBPS and increasing nocturnal blood pressure decline without increasing adverse effects. 


\section{Abbreviations}

ABPM: Ambulatory blood pressure monitoring; MBPS: Morning blood pressure surge; RCT: Randomized control trials; $\beta$-blockers: Adrenergic beta-antagonists; CCB: Calcium channel blockers; RASI: Renin-angiotensin system inhibitors; SBP: Systolic blood pressure; DBP: Diastolic blood pressure; SD: Standard deviation; RR: Relative risk ratios; GITS: Gastrointestinal therapeutic system.

\section{Supplementary Information}

The online version contains supplementary material available at https://doi. org/10.1186/s12872-021-02081-8.

Additional file 1. Search strategy, description of MBPS and supplementary results of meta-analysis.

\section{Acknowledgements}

The authors would like to appreciate Prof Yu Jiang due to his professional assistance in the search strategy and data analysis of article.

\section{Authors' contributions}

$X Y$ designed, supervised, evaluated and approved the manuscript. ZX searched the literature, acquired data, analyzed the results and drafted the manuscript. JZ participated in acquisition of data. CW made critical revisions to this manuscript. All authors approved the final version of the manuscript.

\section{Funding}

This study had no funding support.

\section{Availability of data and materials}

All data generated or analyzed during this study are included in this article and additional information files.

\section{Declarations}

Ethics approval and consent to participate

Not applicable.

\section{Consent for publication}

Not applicable.

\section{Competing interests}

The authors declare that they have no competing interests.

Received: 2 December 2020 Accepted: 25 May 2021

Published online: 04 June 2021

\section{References}

1. Polonsky TS, Bakris GL. Ambulatory blood pressure monitoring. JAMA. 2018;320(17):1807-8.

2. Kario K. Nocturnal hypertension: new technology and evidence. Hypertension (Dallas, Tex: 1979). 2018;71(6).

3. Drawz PE, Alper AB, Anderson AH, Brecklin CS, Charleston J, Chen J, et al. Masked hypertension and elevated nighttime blood pressure in CKD: prevalence and association with target organ damage. Clin J Am Soc Nephrol. 2016;11(4):642-52.

4. Sogunuru GP, Kario K, Shin J, Chen C-H, Buranakitjaroen P, Chia YC, et al. Morning surge in blood pressure and blood pressure variability in Asia: evidence and statement from the HOPE Asia network. J Clin Hypertens (Greenwich). 2019;21(2):324-34.

5. Li Y, Thijs L, Hansen TW, Kikuya M, Boggia J, Richart T, et al Prognostic value of the morning blood pressure surge in 5645 subjects from 8 populations. Hypertension. (Dallas, Tex: 1979). 2010;55(4):1040-8.

6. Amici A, Cicconetti P, Sagrafoli C, Baratta A, Passador P, Pecci T, et al. Exaggerated morning blood pressure surge and cardiovascular events. A 5-year longitudinal study in normotensive and well-controlled hypertensive elderly. Arch Gerontol Geriatr. 2009;49(2):e105-e9.
7. Metoki H, Ohkubo T, Kikuya M, Asayama K, Obara T, Hashimoto J, et al. Prognostic significance for stroke of a morning pressor surge and a nocturnal blood pressure decline: the Ohasama study. Hypertension (Dallas, Tex: 1979). 2006;47(2):149 - 54.

8. Kario K, Pickering TG, Umeda Y, Hoshide S, Hoshide Y, Morinari M, et al. Morning surge in blood pressure as a predictor of silent and clinical cerebrovascular disease in elderly hypertensives: a prospective study. Circulation. 2003;107(10):1401-6.

9. Sheppard JP, Hodgkinson J, Riley R, Martin U, Bayliss S, McManus RJ. Prognostic significance of the morning blood pressure surge in clinical practice: a systematic review. Am J Hypertens. 2015;28(1):30-41.

10. Salles GF, Reboldi G, Fagard RH, Cardoso CR, Pierdomenico SD, Verdecchia P, et al. Prognostic effect of the nocturnal blood pressure fall in hypertensive patients: the ambulatory blood pressure collaboration in patients with hypertension (ABC-H) meta-analysis. Hypertension. 2016;67(4):693-700.

11. Cardoso CRL, Salles GF. Associations of the nocturnal blood pressure fall and morning surge with cardiovascular events and mortality in individuals with resistant hypertension. J Hypertens. 2021.

12. Smolensky MH. Chronobiology and chronotherapeutics. Applications to cardiovascular medicine. Am J Hypertens. 1996;9(4 Pt 3):11S-21S.

13. Hermida RC, Ayala DE, Mojon A, Fernandez JR. Bedtime dosing of antihypertensive medications reduces cardiovascular risk in CKD. J Am Soc Nephrol. 2011;22(12):2313-21.

14. Zhao P, Xu P, Wan C, Wang Z. Evening versus morning dosing regimen drug therapy for hypertension. Cochrane Database Syst Rev. 2011(10):CD004184.

15. Stergiou GS, Nasothimiou EG. Does dosing antihypertensive drugs at night alter renal or cardiovascular outcome: do we have the evidence? Curr Opin Nephrol Hypertens. 2008;17(5):464-9.

16. Hermida RC, Ayala DE, Smolensky MH, Portaluppi F. Chronotherapy in hypertensive patients: administration-time dependent effects of treatment on blood pressure regulation. Expert Rev Cardiovasc Ther. 2007;5(3):463-75.

17. Ezeugo U, Glasser SP. Clinical benefits versus shortcomings of diltiazem once-daily in the chronotherapy of cardiovascular diseases. Expert Opin Pharmacother. 2009;10(3):485-91.

18. Acelajado MC, Pisoni R, Dudenbostel T, Oparil S, Calhoun DA, Glasser SP. Both morning and evening dosing of nebivolol reduces trough mean blood pressure surge in hypertensive patients. J Am Soc Hypertens. 2012;6(1):66-72.

19. Zappe DH, Crikelair N, Kandra A, Palatini P. Time of administration important? Morning versus evening dosing of valsartan. J Hypertens. 2015;33(2):385-92.

20. Zhang Z, Zhang Z, Liu M, Che M, Cao F, Wang R, et al. Influence of different time treatment regimen of antihypertensive medication on morning blood pressure surge and blood pressure variability in hypertension patients. Med J West China. 2014;26(2):221-3 + 6 .

21. Zhao L, Liu S, Gan B. Effect of different time of taking medicine on blood pressure of elderly patients with non-dipper hypertension. Occup Health. 2015;31(13):1794-7 + 801.

22. Qiao Z, Zhuang R, Li Y. Effect of candesartan taken before going to bed on morning blood pressure surge and $\mathrm{MALB}$ in hypertensive patients. Chin J Geriatric Heart Brain Vessel Dis. 2015;17(5):492-4.

23. Li H, Wang D. Intervention effect of enalapril folic acid tablets on $\mathrm{H}$-type hypertensive patients with morning peak blood pressure. Pract J Cardiac Cereb Pneumal Vasc Dis. 2016;24(S1):21-3.

24. Lai J. The clinical effect of chronotherapeutics on morning blood pressure surge in patients with hypertension. J Trop Med. 2015;15(11):1495-8.

25. Peng G, Wang Y, Xiao Y, Chen J, Yang Y, Ye Y, et al. Blood pressure lowering efficacy of telmisartan and amlodipine taking on the morning or at bedtime: ABPM results. Chin J Cardiol. 2013;41(6):484-7.

26. Hermida RC, Ayala DE, Mojon A, Alonso I, Fernandez JR. Reduction of morning blood pressure surge after treatment with nifedipine GITS at bedtime, but not upon awakening, in essential hypertension. Blood Press Monit. 2009;14(4):152-9.

27. Hermida RC, Ayala DE, Mojón A, Fernández JR. Bedtime ingestion of hypertension medications reduces the risk of new-onset type 2 diabetes: a randomised controlled trial. Diabetologia. 2016;59(2):255-65.

28. Hermida RC, Ayala DE, Mojón A, Fernández JR. Risk of incident chronic kidney disease is better reduced by bedtime than 
upon-awakening ingestion of hypertension medications. Hypertens Res. 2018:41(5):342-53.

29. Wang C, Ye Y, Liu C, Zhou Y, Lv L, Cheng C, et al. Evening versus morning dosing regimen drug therapy for chronic kidney disease patients with hypertension in blood pressure patterns: a systematic review and metaanalysis. Intern Med J. 2017;47(8):900-6.

30. Hermida RC, Crespo JJ, Dominguez-Sardina M, Otero A, Moya A, Rios $M T$, et al. Bedtime hypertension treatment improves cardiovascular risk reduction: the Hygia Chronotherapy Trial. Eur Heart J. 2019.

31. Ben-Dov IZ, Kark JD, Ben-Ishay D, Mekler J, Ben-Arie L, Bursztyn M. Predictors of all-cause mortality in clinical ambulatory monitoring: unique aspects of blood pressure during sleep. Hypertension. 2007:49(6):1235-41.

32. Fagard RH, Celis H, Thijs L, Staessen JA, Clement DL, De Buyzere ML, et al. Daytime and nighttime blood pressure as predictors of death and cause-specific cardiovascular events in hypertension. Hypertension 2008:51(1):55-61.

33. Hermida RC, Rios MT, Crespo JJ, Moya A, Dominguez-Sardina M, Otero A, et al. Treatment-time regimen of hypertension medications significantly affects ambulatory blood pressure and clinical characteristics of patients with resistant hypertension. Chronobiol Int. 2013;30(1-2):192-206.

34. Schillaci G, Battista F, Settimi L, Schillaci L, Pucci G. Antihypertensive drug treatment and circadian blood pressure rhythm: a review of the role of chronotherapy in hypertension. Curr Pharm Des. 2015;21 (6):756-72

35. Ramsay LE, ul Haq I, Yeo WW, Jackson PR. Interpretation of prospective trials in hypertension: do treatment guidelines accurately reflect current evidence? J Hypertens Suppl. 1996;14(5):187-S94.

36. Booth JN, Anstey DE, Bello NA, Jaeger BC, Pugliese DN, Thomas SJ, et al. Race and sex differences in asleep blood pressure: The Coronary Artery Risk Development in Young Adults (CARDIA) study. J Clin Hypertens (Greenwich). 2019;21(2):184-92.
37. Booth JN, Jaeger BC, Huang L, Abdalla M, Sims M, Butler M, et al. Morning blood pressure surge and cardiovascular disease events and all-cause mortality in blacks: The Jackson Heart Study. Hypertension. 2020;75(3):835-43.

38. Bombelli M, Fodri D, Toso E, Macchiarulo M, Cairo M, Facchetti R, et al. Relationship among morning blood pressure surge, 24-h blood pressure variability, and cardiovascular outcomes in a white population. Hypertension. 2014;64(5):943-50.

39. Hoshide S, Kario K, de la Sierra A, Bilo G, Schillaci G, Banegas JR, et al. Ethnic differences in the degree of morning blood pressure surge and in its determinants between Japanese and European hypertensive subjects: data from the ARTEMIS study. Hypertension. 2015;66(4):750-6.

40. Meredith PA, Elliott HL. Clinical pharmacokinetics of amlodipine. Clin Pharmacokinet. 1992;22(1):22-31.

41. Pedersen OL, Mancia G, Pickering T, Høegholm A, Julius S, Kjeldsen SE, et al. Ambulatory blood pressure monitoring after 1 year on valsartan or amlodipine-based treatment: a VALUE substudy. J Hypertens. 2007;25(3):707-12.

42. Khodadoustan S, Nasri Ashrafi I, Vanaja Satheesh K, Kumar C, Hs S. S C. Evaluation of the effect of time dependent dosing on pharmacokinetic and pharmacodynamics of amlodipine in normotensive and hypertensive human subjects. Clin Exp Hypertens. 2017;39(6):520-6.

43. Fujimura A, Shiga T, Ohashi K, Ebihara A. Chronopharmacology of amlodipine in rats. Life Sci. 1993;53(7):595-602.

\section{Publisher's Note}

Springer Nature remains neutral with regard to jurisdictional claims in published maps and institutional affiliations.
Ready to submit your research? Choose BMC and benefit from:

- fast, convenient online submission

- thorough peer review by experienced researchers in your field

- rapid publication on acceptance

- support for research data, including large and complex data types

- gold Open Access which fosters wider collaboration and increased citations

- maximum visibility for your research: over $100 \mathrm{M}$ website views per year

At BMC, research is always in progress.

Learn more biomedcentral.com/submissions 